Discussion Expected and unexpected are words that relate to this project. Expected reductions in anxiety where met. One of the most surprising things (needing further research) is where participants reported improvements in physical awareness and health as a result of meditation practice.

Conclusion Meditation (mindfulness) offers a model of how certain hospice skills are eminently transferable to different settings enhancing the hospices model of care, community engagement and profile.

\section{P43 THE USE OF SOCIAL MEDIA BY UK HOSPICES: REVIEW AND DISCUSSION}

${ }^{1}$ Eleanor Kleszzz, ${ }^{1,2}$ Carolyn Campbell. ${ }^{1}$ Cornwall Hospice Care, St Austell, UK, ${ }^{2}$ Peninsula College of Medicine and Dentistry, Plymouth, UK

\subsection{6/bmjspcare-2013-000591.65}

Background With more than $65 \%$ of adults actively using social media each week it is important to ensure that hospices are developing new ways to engage with this population group to encourage fundraising and provide support to patients and carers. Social media provide exciting opportunities for hospices to widen their influence and activity, but also raise practical and ethical issues which must be addressed.

Methods This research appraised 150 hospice websites in England and Wales to determine the prevalence of social media and how it is being used to engage, educate and support hospice users, volunteers and staff. Numbers of users and type of use (fundraising, support, awareness raising) were recorded and factors such as prominence on website explored. Social media sites were also searched for links to hospice and palliative care issues. Results In total, 35\% of hospice websites used Facebook and $33 \%$ used Twitter to communicate with the public, 28\% of websites accessed did not use any form of social media. The majority of social media sites were used to encourage and provide information regarding fundraising. Very few websites used a forum, an opportunity for hospices to provide support to hospice users whilst also educating and encouraging fundraising in a more secure environment. The extended search of social media sites highlighted areas of discussion, support and awareness raising distinct from and not tapped into by hospice sites.

Discussion The rewards and disadvantages of forum use for hospices are explored alongside recommendations for improving public awareness of the hospice social media sites. To date, UK hospices are underusing social media both in volume of traffic and scope (overwhelmingly for fundraising only). This leaves hospices self-excluding from much of the potential of social media to inform, support and debate, and at risk of being left behind.

\section{Education, training and research}

\section{P44 THE INTRODUCTION OF A PATIENT EDUCATION PROGRAMME INTO A HOSPICE DAY SERVICE}

Chris Benson. St. Peter's Hospice, Bristol, UK

\subsection{6/bmjspcare-2013-000591.66}

At a time when hospice care is being scrutinised by commissioners this small study evaluates the implementation of an education programme in a Day Hospice and the impact this had on patients living with life limiting illness.
Education for patients near end of life appears to have received minimal research attention. Wider investigation of the needs of these patients reveals that the impact of illness robs them of their sense of self and autonomy (Johnston 2004), and opportunities to self-care and self-manage can be limited by paternalism (Cottrell 2008, Jackson 2006).

This programme offered patients the opportunity to attend facilitated educational discussion groups such as medicine management, coping with anxiety and breathlessness, and planning ahead for death and to participate in group relaxation and exercise sessions

A case study methodology allowed the use of mixed methods to collect data from three sources; patients, using diaries and transcribed interviews; staff delivering the programme using a focus group; and professionals referring to the service using a questionnaire.

Findings revealed that patients had motivation and capacity to participate and learn however ill they were, demonstrating their desire to be acknowledged as 'active and participating citizens' (Kendall et al 2007:524). Patients also experienced an increase in self-esteem and confidence resulting from the programme.

Staff delivering the programme reported an increase in their confidence through facilitating discussion groups and were challenged in relation to their preconceived ideas about what individual patients may want to learn.

Other findings related to the ability to promote the service to a wider group of potential patients.

This small study demonstrates the potential for increased 'self-care' when an education programme is introduced within a Day Hospice setting. This may positively influence potential commissioners to the service and raise the profile of Day Services as part of Hospice Care.

\section{P45 COMMUNICATING WITH CARE, IN AN EVER INCREASINGLY COMPLEX WORLD}

Janet Dunphy. Kirkwood Hospice, Huddersfield, England

10.1136/bmjspcare-2013-000591.67

Background Within the last decade, numerous white papers have been published to improve care for palliative care patients. The principle of effective communication is at the heart of clinical practice and professional codes. Often communication training programmes are expensive, elitist or inaccessible. It is a challenge to develop a workforce in the current climate and demands of biological and sociological developments.

The more complex healthcare becomes, the more important it becomes to keep it simple.

Aim To improve the confidence and competence of the workforce who will communicate with palliative care patients.

Approach Design \& delivery of innovative, accessible, multiprofessional education. The two day programme includes pre and post training interviews in order to provide meaningful, individual assessment and feedback. The teaching styles are both negotiated and didactic. The programme provides an opportunity for professionals to observe and participate in scenarios which demonstrate key and difficult issues involved in communication. The teaching team consists of 'Jobbing experts' who have undergone advanced communication skills training, specialist clinicians who share the many and various scripts that lie in their memories. Formative assessments include a reflective essay, 
which fits well with portfolio learning/evidence for all professionals including General practitioners.

Outcomes The evaluations have been excellent from all professionals in all clinical arenas. Funding has been secured for further education and collaboration in education is essential to future delivery. The practical element of ensuring information follows the patient, and how to start, support and close conversations were particularly appreciated.

Application to hospice practice Exciting innovative education can embrace key contemporary issues and develop and inspire a workforce Clinical education in the 21st Century must be in partnership and make the complex simple. Negotiated learning by 'telling the story' is both authentic and inspirational. Our stories are worthwhile, inspiring future practitioners - crucial.

\section{P46 THE HOSPICE AND ADULT EDUCATION - A COLLABORATIVE APPROACH}

Carolyn Skilton, Susan Stocks, C Twomey. St Wilfrid's Hospice (Eastbourne), Eastbourne, UK; The Hospice and Adult Education - A collaborative approach

\subsection{6/bmjspcare-2013-000591.68}

Introduction and Aim An initiative with a further education college, a University and local employers identified a need for formal training within end of life care within the care sector. The hospice collaborated with the college to develop an accredited Foundation degree module worth 10 credits targeted at healthcare assistants with a level 3 qualification. Knowledge and skills development and promotion of working in the care sector were key aims.

Method Interviews and questionnaires were completed by the college with local nursing homes and care agencies to assess optimum delivery methods and fee structure. The module content was developed by the hospice which included blended learning, work shadowing and written assignments. Delivery and assessment of the module was undertaken at the hospice. The local college provided administration and study support and collaborated with the University for accreditation.

Results Six students were recruited and successfully completed the programme instigating changes in practice within their organisation. Evaluations were positive from the students, the college and the University. Staff valued the opportunity to study with the hospice and to experience putting theory into practice, whilst gaining an accredited qualification.

Discussion The strength of the programme was local collaboration with the hospice and college. An unexpected outcome was the recruitment of 2 Registered Nurses who used it as a stepping stone for further study. The low module recruitment numbers were disappointing but reflect issues around funding and study leave for staff working in this sector.

Conclusion This project demonstrated a closer relationship between the hospice and the local health and social care community and promoted the hospice as resource and support for end of life care.

\section{P47 INTER PROFESSIONAL PRACTICE PLACEMENTS IN A HOSPICE SETTING}

Gill Thomas. Princess Alice Hospice, Esher, Uk

\subsection{6/bmjspcare-2013-000591.69}

Background Palliative care patients can have complex multidimensional needs. A cohesive multidisciplinary team is vital in the provision of quality care. To achieve this, healthcare professionals need to work alongside and respect each other's professional roles. The use of interprofessional practice placement (IPP) for under graduates has been found to increase understanding of their own role and that of their team colleagues ${ }^{1}$.

Princess Alice Hospice has been supporting IPP placements since 2008. Final year mixed professional students undergo an induction programme and work together on an in-patient palliative care unit.

Aims To explore

- how working together challenges stereotypical views and the impact of this on communication

- the influence of IPP on students ability to communicate with patients and carers

- if IPP equips healthcare students through reflective practice

- observations of team dynamic, confidence and behaviour

Methods The triangulation of course evaluations facilitator feedback and coordinator reflection.

Results As professionals setting out on their career, the students acknowledged their need to appreciate and respect what each profession brings and team working. They found a hospice a challenging place in which to learn but equally learned the value of good communication and psychosocial support for their patients. Students also appreciated the need to be able to communicate effectively with other professions in order to provide patient centred multidisciplinary care.

Conclusion If we are to produce healthcare professionals who will provide a quality health service, they need to speak together and function well as a team. IPP provides the forum for experience and emotional intelligence to be nurtured. Supportive quality healthcare is not just knowing the facts but hinges on effective communication when compassion can be expressed.

Application to hospice practice IPP in a hospice setting is an appropriate place to support novice healthcare professionals in multidisciplinary working and quality care.

\section{P48 BUT WHAT HAPPENS IN A HOSPICE? INTERPROFESSIONAL LEARNING IN THE WORKPLACE}

Sue Taplin. LOROS the Leicestershire and Rutland Hospice, Leicester, UK

\subsection{6/bmjspcare-2013-000591.70}

Building on established links between the University of Leicester Medical School, the School of Social Work and LOROS the Leicestershire Hospice, an interprofessional learning module has been developed which enables social work students on the MA programme at the University of Leicester to experience two days in the life of a Hospice.

This programme, which has now been running for five years, gives social work students the opportunity to meet and learn from different members of the hospice mutidisciplinary team, through a variety of means, including group work, question and answer sessions, meeting informal carers who talk about their experiences of looking after someone at the end of life and a guided tour of the hospice. Although the programme can vary from year to year according to the availability of different members of staff, there is always a session on the role of the social worker in end-of-life care, with teaching on handling difficult situations, exploring the meaning to individuals of loss and change and managing your own emotions in this sensitive and emotional area of practice. 